\title{
Short and long term gene expression variation and networking in human proximal tubule cells when exposed to cadmium
}

\author{
Scott H Garrett ${ }^{1}$, Kaitlin Clarke², Donald A Sens ${ }^{1}$, Youping Deng ${ }^{3}$, Seema Somji ${ }^{1}$, Ke K Zhang ${ }^{1,2^{*}}$ \\ From The 2011 International Conference on Bioinformatics and Computational Biology (BIOCOMP'11) \\ Las Vegas, NV, USA. 18-21 July 2011
}

\begin{abstract}
Cadmium $\left(\mathrm{Cd}^{2+}\right)$ is a known nephrotoxin causing tubular necrosis during acute exposure and potentially contributing to renal failure in chronic long-term exposure. To investigate changes in global gene expression elicited by cadmium, an in-vitro exposure system was developed from cultures of human renal epithelial cells derived from cortical tissue obtained from nephrectomies. These cultures exhibit many of the qualities of proximal tubule cells. Using these cells, a study was performed to determine the cadmium-induced global gene expression changes after short-term ( 1 day, 9, 27, and $45 \mu \mathrm{M}$ ) and long-term cadmium exposure (13 days, 4.5, 9, and $27 \mu \mathrm{M})$. These studies revealed fundamental differences in the types of genes expressed during each of these time points. The obtained data was further analyzed using regression to identify cadmium toxicity responsive genes. Regression analysis showed 403 genes were induced and 522 genes were repressed by $\mathrm{Cd}^{2+}$ within 1 day, and 366 and 517 genes were induced and repressed, respectively, after 13 days. We developed a gene set enrichment analysis method to identify the cadmium induced pathways that are unique in comparison to traditional approaches. The perturbation of global gene expression by various $\mathrm{Cd}^{2+}$ concentrations and multiple time points enabled us to study the transcriptional dynamics and gene interaction using a mutual information-based network model. The most prominent network module consisted of INHBA, KIF20A, DNAJA4, AKAP12, ZFAND2A, AKR1B10, SCL7A11, and AKR1C1.
\end{abstract}

\section{Introduction}

Cadmium is a toxic heavy metal that is widely distributed due to industrial pollution such as mining, refining of metals, burning of fossile fuels, battery production and the use of tobacco. The health threats presented by cadmium has been increasingly recognized by government agencies, healthcare providers and the general public [1]. One of the primary target organs of cadmium toxicity is the human kidney where cadmium accumulates and induces a number of adverse events. The proximal tubule is the main site for cadmium accumulation and the site of cadmium induced toxicity. When the levels of cadmium exceed a tolerance level of the intracellular defense systems, such as

\footnotetext{
* Correspondence: ke.zhang@med.und.edu

'Department of Pathology, School of Medicine and Health Sciences,

University of North Dakota, Grand Forks, ND 58202, USA Full list of author information is available at the end of the article
}

metallothionein and glutathione, cells become susceptive to the toxic effect of this heavy metal. [2,3]. Under high dose acute exposure, cadmium is known to cause proximal tubule necrosis. On the other hand, a few cohort studies showed chronic low doses of cadmium can also induce proximal tubule toxicity $[4,5]$. Toxicokinetic models indicated that the half-life of cadmium in humans is between 15 and 20 years [6]. This suggests that low level exposure of cadmium may be harmful to human kidney via the long term accumulation of this metal in proximal tubule. In this study, we investigate the acute and chronic effects of cadmium on human proximal tubule (HPT) cells. The HPT cell culture system developed in our lab has been shown to have important properties that resemble human proximal tubule [7]. The ability of forming dome by confluent cell monolayers implies the HPT cells retain the vectorial transport activity. The cells are mortal and

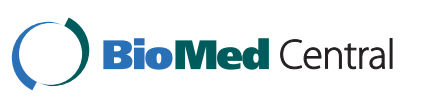


exposure to high concentration of cadmium induces a necrotic mechanism of cell death $[8,9]$. By exposing HPT cells to various doses of cadmium in either a short period of 1 day or a long period of 13 days, we expect different physiological responses to be elicited which represent the acute and chronic responses to cadmium toxicity. In order to investigate the underlying molecular mechanisms of cell toxicity and compensatory cellular defenses related to cadmium exposure, we profiled the global gene expression in HPT cells exposed to increasing concentrations of this metal during short-term (1 day) and long-term (13 days) exposure.

An initial analysis of the microarray data has previously reported a number of genes that were differentially expressed between cadmium treated and control samples [10]. In this manuscript, we investigated how genes respond to different doses of cadmium and how the gene network is formed in response to cadmium toxicity. The high cost of microarray chips prevents researchers from collecting microarray data with large sample sizes; thereby, limiting the power of statistical analysis. In this study, we only have one sample for each condition-time point, however, the overall experimental design enabled us to investigate the gene expression profiles correlated with cadmium concentration. Furthermore, we developed a gene set enrichment analysis (GSEA) method that can be applied to regression-based tests for individual genes, while traditional GSEA methods focus on two-sample comparison analysis, such as $\mathrm{t}$ test.

This study adopted a complicated design by perturbing HPT cells with a variety of doses of cadmium and under various time periods that resulted in variation and dynamics of global gene expression. Therefore, it provides a unique opportunity to investigate the dynamic change of gene expression levels and the network structure of gene interactions in a well-designed experimental environment. A mutual information-based network model [11] was used to investigate the gene networking in response to cadmium toxicity and a network module with a small set of activated genes with high interaction between each other was identified.

\section{Materials and methods}

\section{Cell culture and total RNA collection}

The HPT cell culture system and cadmium treatment has been described previously $[7,10]$. RNA was purified from triplicate cultures of HPT cells by the RNeasy Mini kit (Qiagen, Valencia, CA). The integrity and quality of RNA was ensured via the ribosomal bands using agarose gel electrophoresis.

\section{Microarray analysis}

Microarray experiment was performed by Genome Explorations Inc. (Memphis, TN) using Affymetrix
GeneChip Human Genome U133 Plus 2.0 arrays, which cover over 47,000 human transcripts and variants representing approximately 39,000 of the best characterized human gene transcripts and expressed sequence tags (ests) (Affymetrix, Santa Clara, CA). The scanned images were analyzed using programs resident in GeneChip Operating System v1.4 (GCOS; Affymetrix). The raw data was processed and normalized using MAS 5.0 statistical algorithm that gave detection call regarding signal present or absent for each probe. The correlation of gene expression with cadmium concentrations was assessed using linear regression. The gene signal pathways that were activated by cadmium were identified using gene set enrichment analysis (GSEA) [12]. The $R$ package GSA that was based on standardized statistics was employed for gene set enrichment analysis [13]. The gene list for each pathway was obtained from the Kyoto Encyclopedia of Genes and Genomes (KEGG) http://www.genome.jp/kegg. Cluster analysis was used to group the microarray samples using hierarchical clustering method. The distances between samples were calculated by Pearson dissimilarity and the agglomeration of clusters was performed by Ward linkage method.

\section{Networks analysis}

The relations between genes were defined by mutual information [11]. The resulting adjacent matrix was used for networks analysis. Network modules were identified by hierarchical clustering. The set of genes that have short distances between each other and large distance with other genes was defined as a network module. The most prominent network module was identified for further investigation. All data analyses were performed using $\mathrm{R}$ statistical programming language.

\section{Results}

Gene expression variation in response to acute (1 day) cadmium toxicity

The HPT cells were exposed to various levels of concentrations of $\mathrm{Cd}^{2+}$ for a short term of 24 hours. The $\mathrm{Cd}^{2+}$ concentrations were $0,9,27$, and $45 \mu M$. Our hypothesis is that $\mathrm{Cd}^{2+}$ exposure will elicit an acute toxicity response to HPT cells within one day. As reported before, the HPT cells exhibited a stress response during 24 hour exposure to increasing concentrations of $\mathrm{Cd}^{2+}$ and showed visible signs of toxicity at the high dose [10]. The goal of the current study is to discover the genes that are responsive to the short-term exposure of $\mathrm{Cd}^{2+}$ using messenger RNA microarray data. For each $\mathrm{Cd}^{2+}$ concentration, a sample of HPT cells was harvested, total RNA was purified and submitted for microarray analysis. The Affymetrix U133 Plus 2.0 array was used for this analysis. When using MAS 5.0 for testing, we found 28,720 genes that were present in at least one sample. These genes were 
used for subsequent analysis. In order to identify the genes whose expression levels were dependent on $\mathrm{Cd}^{2+}$ concentrations, we conducted regression analysis for correlating gene expression with concentrations. This resulted in 4,036 genes that have significant $\mathrm{P}$ values $(<$ 0.05). However, none of them had a false discovery rate below 0.05 due to the small size of only 4 samples. Figure 1a shows the $\mathrm{P}$ value distribution that was tilted to low values implying that many genes had positive responses to acute $\mathrm{Cd}^{2+}$ toxicity. If only a few genes had positive responses, the $\mathrm{P}$ values would be evenly distributed. Using a P value cutoff of 0.01 , we reported 403 genes that were positively correlated with cadmium concentration (Table 1), and 522 genes that were negatively correlated (Table 2 ). The positively correlated genes in the top list included heat shock genes like HSP40, HSP70 and HSPBAP1, and ion and anion transporters SLC7A11, SLC16A1, SLC20A2, SLC25A37 and SLC7A1. Both the highest positively and negatively correlated genes contained cell cycle arrest and cell death

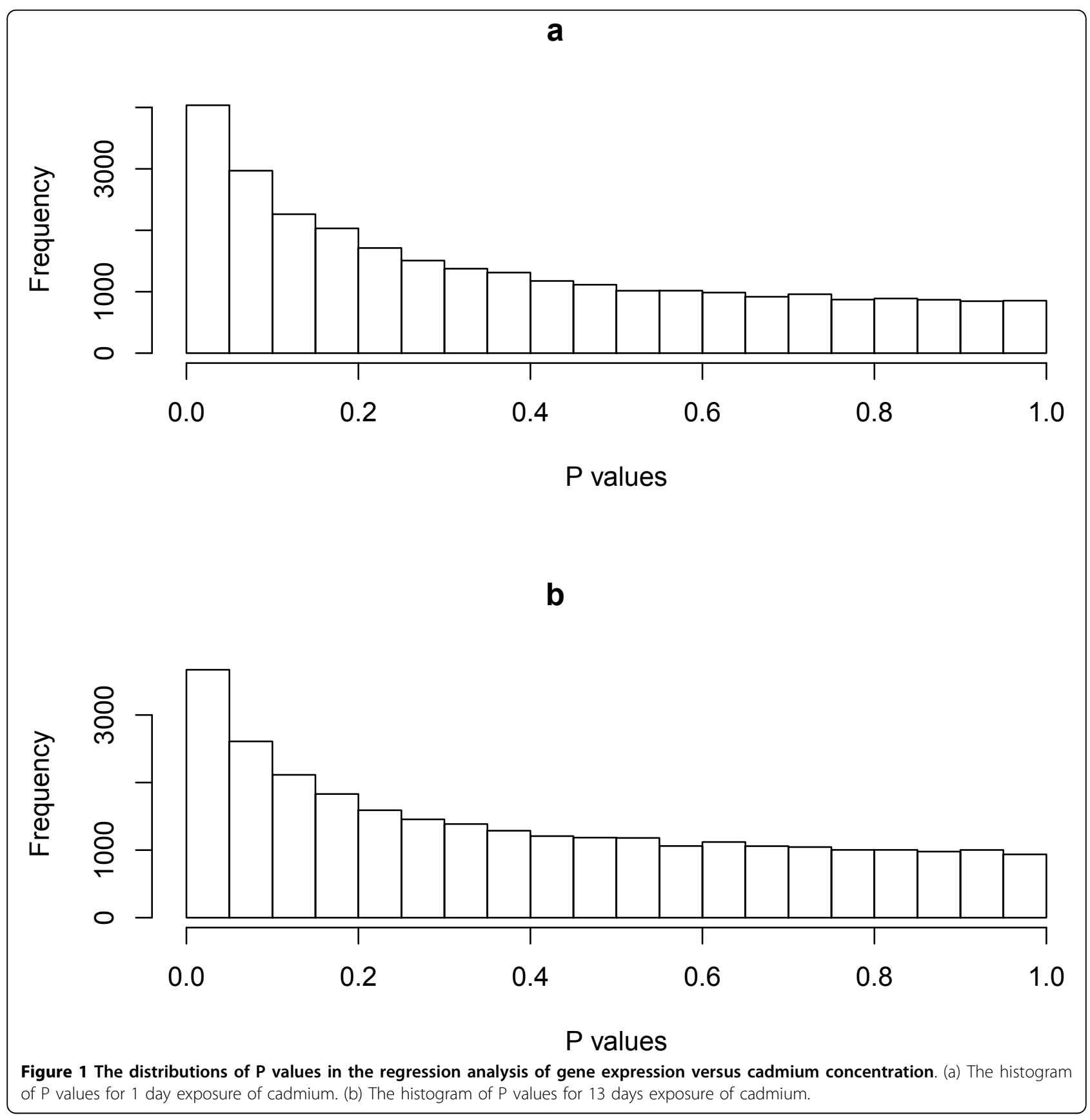


Table $1 \mathrm{Cd}^{2+}$ induced genes in acute cadmium-exposed human proximal tubule cells (1 day, $\mathrm{p}$ value $\left.<0.001\right)$

\begin{tabular}{|c|c|c|c|}
\hline Probe & Symbol & Gene name & $\operatorname{Pr}(>|t|)$ \\
\hline 225061_at & DNAJA4 & DnaJ (Hsp40) homolog, subfamily A, member 4 & 1.16E-05 \\
\hline 207528_s_at & SLC7A11 & solute carrier family 7, (cationic amino acid transporter, y+ system) member 11 & 1.66E-05 \\
\hline 209774_x_at & CXCL2 & chemokine (C-X-C motif) ligand 2 & 4.83E-05 \\
\hline 202744_at & SLC20A2 & solute carrier family 20 (phosphate transporter), member 2 & 5.00E-05 \\
\hline 213524_s_at & GOS2 & G0/G1 switch 2 & 7.15E-05 \\
\hline 220533_at & NA & NA & 7.69E-05 \\
\hline 208544_at & ADRA2B & adrenergic, alpha-2B-, receptor & 7.89E-05 \\
\hline 239211_at & NA & NA & 8.93E-05 \\
\hline 225339_at & SPAG9 & sperm associated antigen 9 & 0.000113 \\
\hline 202235_at & SLC16A1 & solute carrier family 16, member 1 (monocarboxylic acid transporter 1) & 0.000129 \\
\hline 209406_at & BAG2 & BCL2-associated athanogene 2 & 0.000134 \\
\hline 214577_at & MAP1B & microtubule-associated protein 1B & 0.000147 \\
\hline 215404_x_at & FGFR1 & fibroblast growth factor receptor 1 & 0.000153 \\
\hline 229724_at & GABRB3 & gamma-aminobutyric acid (GABA) A receptor, beta 3 & 0.000183 \\
\hline 215034_s_at & TM4SF1 & transmembrane $4 \mathrm{~L}$ six family member 1 & 0.000194 \\
\hline 224809_x_at & TINF2 & TERF1 (TRF1)-interacting nuclear factor 2 & 0.000212 \\
\hline 212223_at & IDS & iduronate 2-sulfatase & 0.000216 \\
\hline 223144_s_at & AKIRIN2 & akirin 2 & 0.000226 \\
\hline 225267_at & KPNA4 & karyopherin alpha 4 (importin alpha 3) & 0.000228 \\
\hline 204186_s_at & PPID & peptidylprolyl isomerase D & 0.000228 \\
\hline 200895_s_at & FKBP4 & FK506 binding protein 4, $59 \mathrm{kDa}$ & 0.000269 \\
\hline 226541_at & $\mathrm{FBXO} 30$ & F-box protein 30 & 0.000307 \\
\hline 204041_at & MAOB & monoamine oxidase B & 0.000372 \\
\hline 1557915_s_at & GSTO1 & glutathione S-transferase omega 1 & 0.000435 \\
\hline 202199_s_at & SRPK1 & SFRS protein kinase 1 & 0.000476 \\
\hline 211696_x_at & HBB & hemoglobin, beta & 0.000491 \\
\hline 224888_at & SELI & selenoprotein I & 0.000593 \\
\hline 227408_s_at & $\mathrm{SN} \times 25$ & sorting nexin 25 & 0.000618 \\
\hline 222703_s_at & YRDC & yrdC domain containing (E. coli) & 0.000654 \\
\hline 229355_at & NA & NA & 0.000654 \\
\hline 200065_s_at & ARF1 & ADP-ribosylation factor 1 & 0.000666 \\
\hline 210260_s_at & TNFAIP8 & tumor necrosis factor, alpha-induced protein 8 & 0.000669 \\
\hline 231629_x_at & KLK3 & kallikrein-related peptidase 3 & 0.000683 \\
\hline 201829_at & NET1 & neuroepithelial cell transforming 1 & 0.000719 \\
\hline 240633_at & DOK7 & docking protein 7 & 0.000724 \\
\hline 225766_s_at & TNPO1 & transportin 1 & 0.00083 \\
\hline 219334_s_at & $\mathrm{OBFC2A}$ & oligonucleotide/oligosaccharide-binding fold containing $2 \mathrm{~A}$ & 0.000846 \\
\hline 227622_at & PCF11 & PCF11, cleavage and polyadenylation factor subunit, homolog (S. cerevisiae) & 0.000872 \\
\hline 217561_at & CALCA & calcitonin-related polypeptide alpha & 0.000891 \\
\hline 214638_s_at & CCNT2 & cyclin T2 & 0.000898 \\
\hline 218624_s_at & MGC2752 & hypothetical LOC65996 & 0.000989 \\
\hline
\end{tabular}

genes such as G0S2, GSPT1 and BAG5. These activated genes represented a stress response for the acute $\mathrm{Cd}^{2+}$ toxicity.

Our next aim was to identify the gene regulatory pathways that were activated in the HPT cells in response to the acute, 1 day exposure to $\mathrm{Cd}^{2+}$. The gene pathway database compiled in Kyoto Encyclopedia of Genes and Genomes (KEGG) has been a valuable source for investigating pathways. The gene set enrichment analysis
(GSEA) method was employed to identify activated KEGG pathways using the microarray data. The approach of GSEA is to test whether the ranks of $P$ values of a set of genes are randomly distributed among all the $\mathrm{P}$ values [12]. Because most of GSEA methods employ two sample comparison method such as unpaired or paired t test [13], we set out to develop a statistical testing method for GSEA based on regression analysis for each individual gene. As described earlier, out of a total of 28,720 genes, 
Table $2 \mathrm{Cd}^{2+}$ repressed genes in acute cadmium-exposed human proximal tubule cells $(1$ day, $p$ value $<0.001)$

\begin{tabular}{|c|c|c|c|}
\hline Probe & Symbol & Gene name & $\operatorname{Pr}(>|t|)$ \\
\hline 213067_at & MYH10 & myosin, heavy chain 10 , non-muscle & 4.01E-05 \\
\hline 225276_at & GSPT1 & G1 to $S$ phase transition 1 & $8.75 \mathrm{E}-05$ \\
\hline 232349_x_at & DCAF6 & DDB1 and CUL4 associated factor 6 & 0.000115 \\
\hline 1557986_s_at & SMCR8 & Smith-Magenis syndrome chromosome region, candidate 8 & 0.000121 \\
\hline 223141_at & UCK1 & uridine-cytidine kinase 1 & 0.000123 \\
\hline 209196_at & WDR46 & WD repeat domain 46 & 0.000125 \\
\hline 227477_at & ZMYND19 & zinc finger, MYND-type containing 19 & 0.000149 \\
\hline 223396_at & TMEM60 & transmembrane protein 60 & 0.00018 \\
\hline 227765_at & NA & NA & 0.000189 \\
\hline 235812_at & TMEM188 & transmembrane protein 188 & 0.000237 \\
\hline 239464_at & NA & NA & 0.000239 \\
\hline 202337_at & PMF1 & polyamine-modulated factor 1 & 0.000242 \\
\hline 1570506_at & NA & NA & 0.000247 \\
\hline 1555630_a_at & RAB34 & RAB34, member RAS oncogene family & 0.000249 \\
\hline 225831_at & LUZP1 & leucine zipper protein 1 & 0.000254 \\
\hline 204027_s_at & METTL1 & methyltransferase like 1 & 0.000295 \\
\hline 212694_s_at & PCCB & propionyl Coenzyme A carboxylase, beta polypeptide & 0.000304 \\
\hline 204104_at & SNAPC2 & small nuclear RNA activating complex, polypeptide 2, $45 \mathrm{kDa}$ & 0.000369 \\
\hline 216308_x_at & GRHPR & glyoxylate reductase/hydroxypyruvate reductase & 0.000392 \\
\hline 233233_at & NA & NA & 0.000438 \\
\hline 200980_s_at & PDHA1 & pyruvate dehydrogenase (lipoamide) alpha 1 & 0.000468 \\
\hline 218042_at & COPS4 & COP9 constitutive photomorphogenic homolog subunit 4 (Arabidopsis) & 0.000476 \\
\hline 210829_s_at & SSBP2 & single-stranded DNA binding protein 2 & 0.000504 \\
\hline 203226_s_at & TSPAN31 & tetraspanin 31 & 0.000516 \\
\hline 202266_at & TTRAP & TRAF and TNF receptor associated protein & 0.000541 \\
\hline 221856_s_at & FAM63A & family with sequence similarity 63, member $A$ & 0.00066 \\
\hline 227224_at & RALGPS2 & Ral GEF with PH domain and SH3 binding motif 2 & 0.000663 \\
\hline 243309_at & FLJ27352 & hypothetical LOC145788 & 0.000673 \\
\hline 202883_s_at & PPP2R1B & protein phosphatase 2 (formerly $2 A$ ), regulatory subunit $A$, beta isoform & 0.000684 \\
\hline 201801_s_at & SLC29A1 & solute carrier family 29 (nucleoside transporters), member 1 & 0.000688 \\
\hline 220974_x_at & SFXN3 & sideroflexin 3 & 0.000699 \\
\hline 1555679_a_at & RTN4IP1 & reticulon 4 interacting protein 1 & 0.000777 \\
\hline 212872_s_at & MED20 & mediator complex subunit 20 & 0.00078 \\
\hline 201888_s_at & IL13RA1 & interleukin 13 receptor, alpha 1 & 0.000787 \\
\hline 227567_at & NA & NA & 0.00079 \\
\hline 229146_at & C7orf31 & chromosome 7 open reading frame 31 & 0.000806 \\
\hline 224779_s_at & FAM96A & family with sequence similarity 96 , member A & 0.000843 \\
\hline 213022_s_at & UTRN & utrophin & 0.000846 \\
\hline 226710_at & C8orf82 & chromosome 8 open reading frame 82 & 0.000846 \\
\hline 213181_s_at & MOCS1 & molybdenum cofactor synthesis 1 & 0.000878 \\
\hline 218268_at & TBC1D15 & TBC1 domain family, member 15 & 0.000883 \\
\hline 211708_s_at & SCD & stearoyl-CoA desaturase (delta-9-desaturase) & 0.000916 \\
\hline 202299_s_at & HBXIP & hepatitis B virus $x$ interacting protein & 0.000948 \\
\hline 213574_s_at & KPNB1 & karyopherin (importin) beta 1 & 0.00097 \\
\hline 205052_at & $\mathrm{AUH}$ & AU RNA binding protein/enoyl-Coenzyme A hydratase & 0.00097 \\
\hline
\end{tabular}

4036 were found to have a significant $\mathrm{P}$ values $(<0.05)$ correlating to $\mathrm{Cd}^{2+}$ concentrations. Suppose a KEGG pathway has $\mathrm{n}$ genes and $\mathrm{x}$ genes are significant using regression analysis. Our GSEA method consists of deriving a hypergemetric probability function shown below.

$$
p(x)=\frac{\left(\begin{array}{c}
4036 \\
x
\end{array}\right)\left(\begin{array}{c}
28720-4036 \\
n-x
\end{array}\right)}{\left(\begin{array}{c}
22000 \\
n
\end{array}\right)}
$$


Using this function, we can test whether $\mathrm{x}$ is significantly larger than what would be expected from a hypergeometric distribution. Statistically, if we observe $r$ genes in the significant set, $\mathrm{P}(\mathrm{X}>\mathrm{r})$ designates the $\mathrm{P}$ value for the significance of $r$ genes. Thus, $P$ value $=P$ $(\mathrm{X}>\mathrm{r})=\mathrm{p}(\mathrm{r}+1)+\mathrm{p}(\mathrm{r}+2)+\mathrm{p}(\mathrm{r}+3)+\ldots+\mathrm{p}(\mathrm{x})$. This gene set enrichment analysis found 3 significant pathways, NOD-like receptor signaling pathway, base excision repair, and steroid biosynthesis, with p values 0.0486 , $0.0385,0.0009$, respectively. NOD-like receptors play an important role in regulation of inflammatory and apoptotic responses and are activated to stress response [14]. DNA repair is a mode of action known to response to heavy metal toxicity. Steroid biosynthesis is a metabolic process that is required for recovery from an acute toxicity attack.

\section{Gene expression variation in response to long term (13 day) cadmium toxicity}

HPT cells were exposed to $\mathrm{Cd}^{2+}$ for a long period in order to determine the mechanism by which global gene expression adapted to environmental challenge. HPT cells were exposed to various concentrations of $\mathrm{Cd}^{2+}, 4.5,9$ and $27 \mu \mathrm{M} \mathrm{Cd}^{+2}$ for 13 days. Lower concentration of $\mathrm{Cd}^{2+}$ were used for the 13 day time course since $45 \mu \mathrm{M} \mathrm{Cd}^{2+}$ caused toxicity and a loss of cell viability. There was no loss of cell viability when the cells were treated with 4.5 and $9 \mu \mathrm{M} \mathrm{Cd}^{2+}$ over a 13 day period, while exposure to $27 \mu \mathrm{M} \mathrm{Cd}^{+2}$ caused an approximate $30 \%$ loss in cell viability [10]. The ability of the HPT cells to form domes was only affected by exposure to $27 \mu \mathrm{M} \mathrm{Cd}^{+2}$ for 13 days. The microarray data were processed and analyzed as described above for 1 day exposure. The distribution of $\mathrm{P}$ values for each individual gene is shown in Figure 1b. The distribution is significantly skewed to small $\mathrm{P}$ values, implying a large number of genes were altered by cadmium exposure. From a total of 28,720 genes, 366 were positively correlated with the cadmium concentration, and 517 were negatively correlated at a $\mathrm{P}$ value cutoff of 0.01 (Table 3 and 4). Similar to that observed for 1 day exposure, long term cadmium exposure altered the expression of a number of cell cycle control and cell death genes, including GSPT1, G2E3 and BCL2. The expression of metal binding and transport genes, such as CAB39L, TMC5, SLC5A3 and SLC35F2, were affected as well. However, expression levels of stress response genes such as heat shock and DNA repair genes were not changed after 13 days exposure, instead, many tumor-related genes were activated. These included RAB42, DEK, RRAD, TPD52L1 and a variety of mitogen related kinases. The GSEA analysis revealed 9 altered KEGG pathways including 3 cancer pathways, prostate cancer, melanoma and p53 signaling pathway (Table 5). None of the pathways overlapped with the ones identified after 24 hours exposure.
The common expression patterns between 1 day and 13 day of cadmium exposure

In order to investigate genes that showed expression variation throughout both short and long term cadmium exposure, we conducted analysis for the pooled data of the two time points. Figure 2 shows a heat map for the 8 samples for 1 day and 13 day exposure. The data were normalized to a grand mean of 0 and only 901 genes with standard deviation $>1$ were used for this analysis. Hierarchical clustering method that was based on Pearson dissimilarity and Ward linkage showed as expected that the HPT cells treated with high doses of $\mathrm{Cd}^{2+}$ tended to be grouped together. The cells exposed to 27 and $45 \mu \mathrm{M} \mathrm{Cd}^{2+}$ for both 1 day and 13 days formed a group, and all cells exposed to $9 \mu \mathrm{M}$ or lower $\mathrm{Cd}^{2+}$ concentration formed the other major group. The change of dissimilarity and linkage methods did not alter the results. There were 17 genes that were positively correlated with cadmium concentration for both 1 day and 13 day exposure (Figure 3a). Given that the number of positively correlated genes was 403 and 366 for 1 and 13 day respectively, the random number of overlapping genes can be modeled as a hypergeometric distribution. The probability of observing more than 17 overlapping genes from the random sets of 403 and 366 genes is $5.89 \mathrm{E}-06$. Thus, the two exposure time periods had a significant number of common genes that were positively correlated with cadmium concentration. Similarly, we found 26 genes that were negative correlated with cadmium concentration for both time periods (Figure $3 \mathrm{~b})$. This number of overlapping genes was statistically significant when compared to selecting 26 gene by chance (P value $=1.32 \mathrm{E}-06$ ). Therefore, we conclude that the HPT cells have a common response component in global gene expression variation during short term and long term cadmium exposure.

\section{Gene regulatory network in response to cadmium exposure}

In response to heavy metal toxicity, thousands of genes will be activated forming a complicated gene regulatory network interacting cooperatively. To decode the complete gene network for cadmium response is impossible for the small sample size used in this study and high complexity of network structure [15]. It is also computational forbidden to construct a network by including a large number of genes. Therefore, we modeled the gene network focusing on only the 901 identified genes as having high variability shown in the heat map. Mutual information for transcriptional levels was used to quantify the relations between genes. The resulting adjacent matrix was analyzed for the presence of an active network module that has high connectivity within the module and low connectivity with other components of the network. Figure 4 shows a network modules consisting of 8 genes. DNAJA4 encodes the 
Table $3 \mathrm{Cd}^{2+}$ induced genes in chronic cadmium-exposed human proximal tubule cells $(13$ days, $\mathrm{p}$ value $<0.001)$

\begin{tabular}{|c|c|c|c|}
\hline Probe & Symbol & Gene name & $\operatorname{Pr}(>|t|)$ \\
\hline 225914_s_at & CAB39L & calcium binding protein 39-like & 1.69E-05 \\
\hline 210372_s_at & TPD52L1 & tumor protein D52-like 1 & 2.73E-05 \\
\hline 211499_s_at & MAPK11 & mitogen-activated protein kinase 11 & 3.07E-05 \\
\hline 222587_s_at & GALNT7 & UDP-N-acetyl-alpha-D galactosamine:polypeptide N-acetylgalactosaminyltransferase 7 (GalNAc-T7) & $6.05 \mathrm{E}-05$ \\
\hline 233528_s_at & GATSL3 & GATS protein-like 3 & 8.85E-05 \\
\hline 212335_at & GNS & glucosamine ( $\mathrm{N}$-acetyl)-6-sulfatase & 9.88E-05 \\
\hline 217748_at & ADIPOR1 & adiponectin receptor 1 & 0.000112 \\
\hline 222601_at & UBA6 & ubiquitin-like modifier activating enzyme 6 & 0.000155 \\
\hline 219349_s_at & EXOC2 & exocyst complex component 2 & 0.000168 \\
\hline 218319_at & PELI1 & pellino homolog 1 (Drosophila) & 0.000213 \\
\hline 203553_s_at & MAP4K5 & mitogen-activated protein kinase kinase kinase kinase 5 & 0.000219 \\
\hline 1560779_a_at & NA & NA & 0.000227 \\
\hline 244056_at & SFTA2 & surfactant associated 2 & 0.000236 \\
\hline 223797_at & PRO2852 & hypothetical protein PRO2852 & 0.000366 \\
\hline 219580_s_at & TMC5 & transmembrane channel-like 5 & 0.000373 \\
\hline 1553367_a_at & COX6B2 & cytochrome c oxidase subunit VIb polypeptide 2 (testis) & 0.000413 \\
\hline 200618_at & LASP1 & LIM and SH3 protein 1 & 0.000414 \\
\hline 203925_at & GCLM & glutamate-cysteine ligase, modifier subunit & 0.000502 \\
\hline 209198_s_at & SYT11 & synaptotagmin XI & 0.000509 \\
\hline 213107_at & TNIK & TRAF2 and NCK interacting kinase & 0.000532 \\
\hline 212341_at & YIPF6 & Yip1 domain family, member 6 & 0.000565 \\
\hline 223799_at & KIAA1826 & KIAA1826 & 0.000598 \\
\hline 228810_at & CCNYL1 & cyclin Y-like 1 & 0.000665 \\
\hline 218472_s_at & PELO & pelota homolog (Drosophila) & 0.000669 \\
\hline 227022_at & GNPDA2 & glucosamine-6-phosphate deaminase 2 & 0.000688 \\
\hline 1552712_a_at & NMNAT2 & nicotinamide nucleotide adenylyltransferase 2 & 0.00069 \\
\hline 217144_at & UBB & ubiquitin B & 0.000699 \\
\hline 1559514_at & LOC100132077 & hypothetical protein LOC100132077 & 0.000707 \\
\hline 1552946_at & ZNF114 & zinc finger protein 114 & 0.000794 \\
\hline 226071_at & ADAMTSL4 & ADAMTS-like 4 & 0.000822 \\
\hline 204802_at & RRAD & Ras-related associated with diabetes & 0.000874 \\
\hline 208816_x_at & ANXA2P2 & annexin A2 pseudogene 2 & 0.00088 \\
\hline 212791_at & C1orf216 & chromosome 1 open reading frame 216 & 0.000882 \\
\hline 215704_at & FLG & filaggrin & 0.000923 \\
\hline 200638_s_at & YWHAZ & tyrosine 3-monooxygenase/tryptophan 5-monooxygenase activation protein, zeta polypeptide & 0.000945 \\
\hline
\end{tabular}

heat shock protein, SLC7A11 an anion amino acid transporter, AKR1B10 and AKR1C1 are subunits for a reductase associated with cancer, ASPM and KIF20A are involved in mitotic spindle functions, ZFAND2A is a zincfinger protein that is inducible by arsenite, and INHBA is known to inhibit cell proliferation and to have tumor-suppressor activity. The network modules provide hypotheses for gene interactions which can be verified in subsequent biological experiments.

\section{Discussion}

Microarray experiments have become a standard approach to simultaneously quantify the transcriptional levels of thousands genes under various conditions. However, due to the high cost of chips, microarray experiments are often carried out with a limited sample size, which requires a careful experimental design. In this study, we chose two time points, 1 day and 13 day, to account for toxicity caused by short and long cadmium exposure. For each time point, we had HPT cells exposed to 3 cadmium concentrations plus a control sample (non-treated). Thus, even with only a single replicate at each concentration, we were able to test the correlation between transcriptional levels and cadmium concentrations. Our analysis suggests that experimental design with various conditions overcomes the difficulty of lack of replications.

Exposure of HPT cells to 1 day and 13 days of cadmium induces distinct sets of genes. At 1 day, HPT cells appeared to have a stress response that activated a number of heat shock and DNA repair genes and pathways. 
Table $4 \mathrm{Cd}^{2+}$ repressed genes in chronic cadmium-exposed human proximal tubule cells (13 days, $\mathrm{p}$ value $<0.001$ )

\begin{tabular}{|c|c|c|c|}
\hline Probe & Symbol & Gene name & $\operatorname{Pr}(>|\mathbf{t}|)$ \\
\hline 218025_s_at & $\mathrm{PECl}$ & peroxisomal D3,D2-enoyl-CoA isomerase & $3.66 \mathrm{E}-05$ \\
\hline 202781_s_at & INPP5K & inositol polyphosphate-5-phosphatase K & $6.81 \mathrm{E}-05$ \\
\hline 216053_x_at & FAM182A & family with sequence similarity 182 , member $\mathrm{A}$ & 0.0001 \\
\hline 228665_at & CYYR1 & cysteine/tyrosine-rich 1 & 0.000109 \\
\hline 228374_at & C10orf28 & chromosome 10 open reading frame 28 & 0.000119 \\
\hline 224913_s_at & TIMM50 & translocase of inner mitochondrial membrane 50 homolog (S. cerevisiae) & 0.000131 \\
\hline 215438_x_at & GSPT1 & G1 to $S$ phase transition 1 & 0.000132 \\
\hline 218404_at & SNX10 & sorting nexin 10 & 0.000133 \\
\hline 226016_at & CD47 & CD47 molecule & 0.000135 \\
\hline 203574_at & NFIL3 & nuclear factor, interleukin 3 regulated & 0.000136 \\
\hline 227190_at & TMEM37 & transmembrane protein 37 & 0.00017 \\
\hline 201225_s_at & SRRM1 & serine/arginine repetitive matrix 1 & 0.000211 \\
\hline 1552846_s_at & RAB42 & RAB42, member RAS oncogene family & 0.000217 \\
\hline 228520_s_at & APLP2 & amyloid beta (A4) precursor-like protein 2 & 0.000219 \\
\hline 227769_at & NA & NA & 0.000231 \\
\hline 201855_s_at & ATMIN & ATM interactor & 0.000256 \\
\hline 219379_x_at & ZNF358 & zinc finger protein 358 & 0.000275 \\
\hline 32811_at & MYO1C & myosin IC & 0.000282 \\
\hline 200707_at & PRKCSH & protein kinase $\mathrm{C}$ substrate $80 \mathrm{~K}-\mathrm{H}$ & 0.000296 \\
\hline 229007_at & LOC283788 & FSHD region gene 1 pseudogene & 0.000301 \\
\hline 219271_at & GALNT14 & UDP-N-acetyl-alpha-D-galactosamine:polypeptide Nacetylgalactosaminyltransferase 14 (GalNAc-T14) & 0.000304 \\
\hline 201816_s_at & GBAS & glioblastoma amplified sequence & 0.000305 \\
\hline 217817_at & ARPC4 & actin related protein $2 / 3$ complex, subunit $4,20 \mathrm{kDa}$ & 0.000315 \\
\hline 201185_at & HTRA1 & HtrA serine peptidase 1 & 0.000316 \\
\hline 205739_x_at & ZNF107 & zinc finger protein 107 & 0.000323 \\
\hline 212418_at & ELF1 & E74-like factor 1 (ets domain transcription factor) & 0.000327 \\
\hline 209431_s_at & PATZ1 & POZ (BTB) and AT hook containing zinc finger 1 & 0.000367 \\
\hline 201549_x_at & KDM5B & lysine (K)-specific demethylase 5B & 0.000373 \\
\hline 203858_s_at & $\operatorname{cox} 10$ & COX10 homolog, cytochrome c oxidase assembly protein, heme A: farnesyltransferase (yeast) & 0.000425 \\
\hline 218483_s_at & C11orf60 & chromosome 11 open reading frame 60 & 0.00044 \\
\hline 218045_x_at & PTMS & parathymosin & 0.000454 \\
\hline 212493_s_at & SETD2 & SET domain containing 2 & 0.000459 \\
\hline 204805_s_at & $\mathrm{H} 1 \mathrm{FX}$ & $\mathrm{H} 1$ histone family, member $\mathrm{X}$ & 0.000471 \\
\hline 226926_at & DMKN & dermokine & 0.000474 \\
\hline 211025_x_at & COX5B & cytochrome c oxidase subunit Vb & 0.000494 \\
\hline 212904_at & LRRC47 & leucine rich repeat containing 47 & 0.000544 \\
\hline 215543_s_at & LARGE & like-glycosyltransferase & 0.000546 \\
\hline 228998_at & TNRC6B & trinucleotide repeat containing 6B & 0.000575 \\
\hline 210220_at & FZD2 & frizzled homolog 2 (Drosophila) & 0.000632 \\
\hline 225247_at & C19orf6 & chromosome 19 open reading frame 6 & 0.00065 \\
\hline 212264_s_at & WAPAL & wings apart-like homolog (Drosophila) & 0.00066 \\
\hline 244287_at & SFRS12 & splicing factor, arginine/serine-rich 12 & 0.000661 \\
\hline 221829_s_at & TNPO1 & transportin 1 & 0.000704 \\
\hline 1553313_s_at & SLC5A3 & solute carrier family 5 (sodium/myo-inositol cotransporter), member 3 & 0.000786 \\
\hline 232412_at & FBXL20 & F-box and leucine-rich repeat protein 20 & 0.000788 \\
\hline 217964_at & TTC19 & tetratricopeptide repeat domain 19 & 0.000788 \\
\hline 223056_s_at & XPO5 & exportin 5 & 0.000792 \\
\hline 213470_s_at & HNRNPH1 & heterogeneous nuclear ribonucleoprotein $\mathrm{H} 1(\mathrm{H})$ & 0.000816 \\
\hline 214543_x_at & QKI & quaking homolog, $\mathrm{KH}$ domain RNA binding (mouse) & 0.00085 \\
\hline 243829_at & BRAF & v-raf murine sarcoma viral oncogene homolog B1 & 0.000864 \\
\hline 34260_at & TELO2 & TEL2, telomere maintenance 2 , homolog (S. cerevisiae) & 0.000874 \\
\hline
\end{tabular}


Table $4 \mathrm{Cd}^{2+}$ repressed genes in chronic cadmium-exposed human proximal tubule cells $(13$ days, $p$ value ?<? 0.001$)$ (Continued)

\begin{tabular}{|c|c|c|c|}
\hline 203392_s_at & CTBP1 & C-terminal binding protein 1 & 0.000877 \\
\hline 201928_at & PKP4 & plakophilin 4 & 0.000888 \\
\hline 232814_x_at & $\mathrm{KLC} 1$ & kinesin light chain 1 & 0.000907 \\
\hline 225399_at & TSEN15 & tRNA splicing endonuclease 15 homolog (S. cerevisiae) & 0.000924 \\
\hline 236035_at & NA & NA & 0.000931 \\
\hline 229400_at & HOXD10 & homeobox D10 & 0.000961 \\
\hline 208847_s_at & $\mathrm{ADH} 5$ & alcohol dehydrogenase 5 (class III), chi polypeptide & 0.000984 \\
\hline 214800_x_at & BTF3 & basic transcription factor 3 & 0.000992 \\
\hline 204619_s_at & VCAN & versican & 0.000997 \\
\hline 218826_at & SLC35F2 & solute carrier family 35 , member $F 2$ & 0.000998 \\
\hline
\end{tabular}

This was expected because stress responses that are characterized with induced expression of heat shock proteins are acute and transient that last for only a few hours to a few days [16-20]. This acute response to $\mathrm{Cd}^{2}$ ${ }^{+}$with the induction of heat shock proteins, such as HSP 27, 60, 70 and 90, have been repeatedly verified in our previous studies [21-24].

The 13 days exposure was used to model the chronic response of kidney to environment of cadmium exposure. The molecular changes in the HPT cells in response to long term cadmium exposure were manifested with activation of many oncogenes and cancer pathways. The association of cancer occurrence and cadmium exposure has been established by numerous cohort studies [25-27]. Although 13 days of exposure might not exactly represent the chronic effect of cadmium on the kidney living environment, our experiment suggests that the cell culture system serves as a valid model for studying the long term toxicity of cadmium on the kidney.

Although the two time periods of cadmium exposure appeared to induce two distinct toxicity responses, the acute and chronic responses, they shared some common features in gene expression. The heat map showed that the samples were not separated by time of exposure, instead, the samples tended to be grouped by doses

Table $5 \mathrm{Cd}^{2+}$ activated KEGG pathways in chronic cadmium-exposed human proximal tubule cells (13 days)

\begin{tabular}{lr}
\hline KEGG Pathway & P value \\
\hline Lysine biosynthesis & 0.000182016 \\
Sulfur metabolism & 0.001924676 \\
Glutathione metabolism & 0.001973874 \\
Caffeine metabolism & 0.004896048 \\
Protein processing in endoplasmic reticulum & 0.022267231 \\
Prostate cancer & 0.035237612 \\
Melanoma & 0.035316953 \\
Valine, leucine and isoleucine degradation & 0.038038497 \\
p53 signaling pathway & 0.041271084 \\
\hline
\end{tabular}

across two time points. All the HPT samples that were treated with $27 \mu \mathrm{M} \mathrm{Cd}^{2+}$ for both 1 day and 13 days formed a cluster. When looking into the genes that were correlated with cadmium concentrations in both 1 day and 13 days, we found that the number of overlapping genes was significantly larger than what was expected from random selection. The set of overlapping genes included ion transporter proteins, kinases, and transcriptional factors. These genes should be further investigated to reveal their functions in toxicity responses.

For any biophysical process, thousands of genes interact to form a network to maintain their biological functions. There are commonly two statistical approaches to study gene interaction, gene set enrichment analysis (GSEA) and networks analysis. GSEA has been successfully applied to microarray data to identify the activated pathways. However, GSEA has been so far focused on comparing different treatment groups, mostly for two groups, treatment and control $[12,13,28,29]$. For this study, we developed a GSEA method that can be used for ranks of $P$ values from any statistical tests. We have showed that our proposed GSEA statistic follows a hypergeometric distribution. Thus, the $\mathrm{P}$ value of the GSEA is available.

Gene networks analysis is computationally challenging. Networks are often highly complicated, consisting of a large number of genes that can be fairly dynamic in nature. Decoding such a gene network requires researchers to quantify the global gene expression under dynamic and variable conditions. The current study provides a unique opportunity in that microarray data was acquired at multiple time points and at various levels of $\mathrm{Cd}^{2+}$ exposure. We used mutual information to account for the relations between genes. Mutual information is able to measure gene dependency without assuming data distributions and linear relationship. However, the mutual information-based network models require huge amount of computation that is not feasible for modeling all the genes. For this reason, we focused on only a set of most 


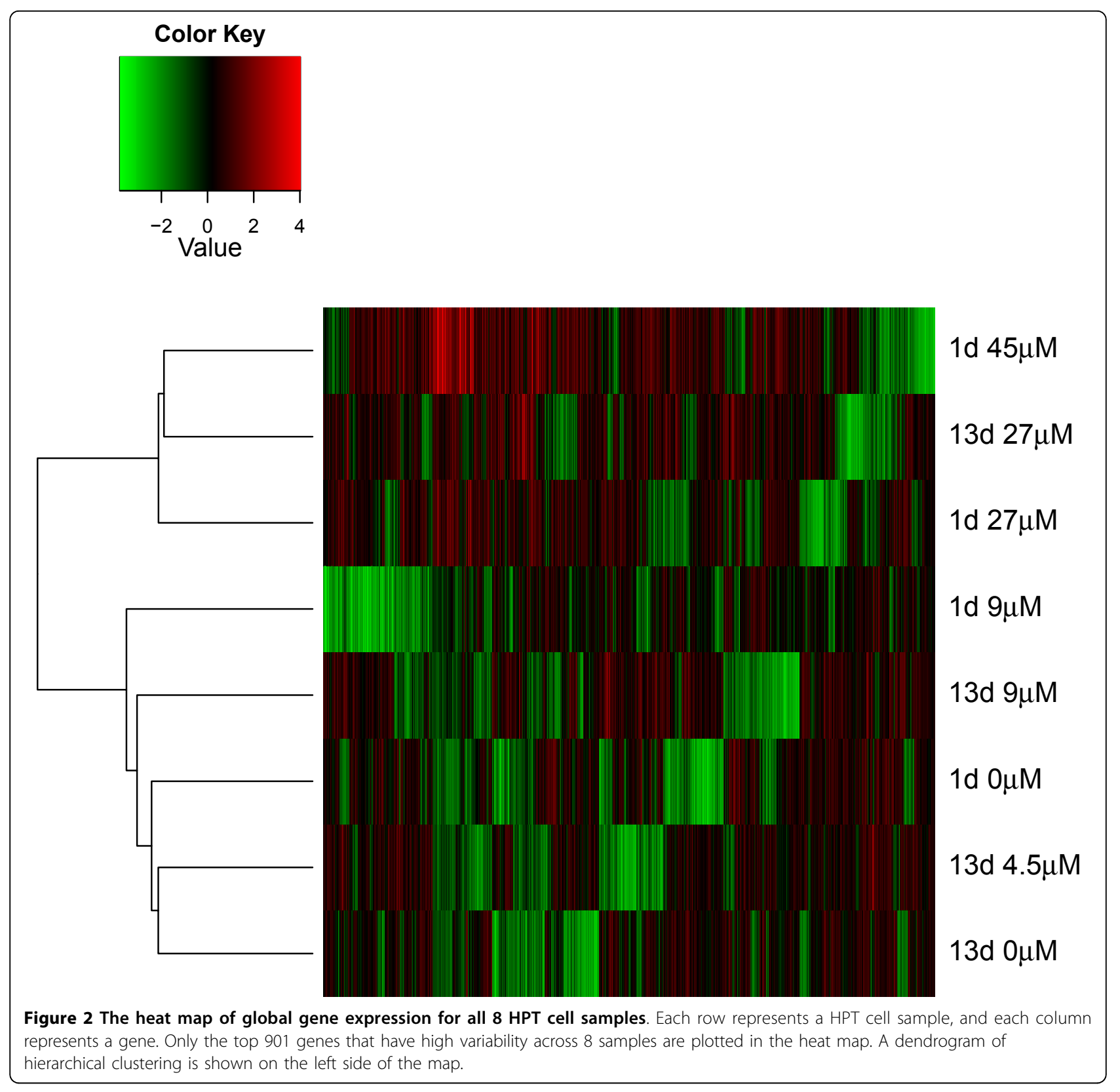

a)

Repressed Genes

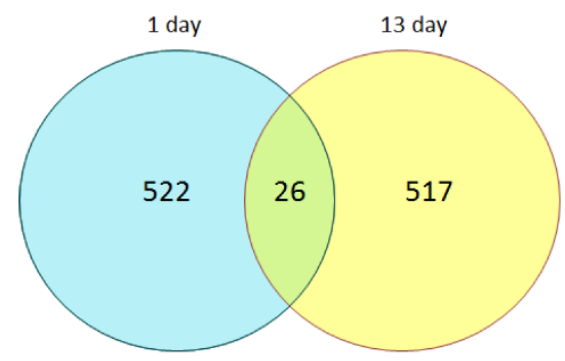

b)

Induced Genes

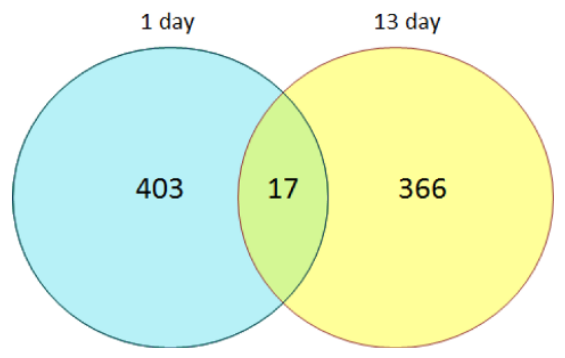

Figure 3 Venn diagram depicting the number of genes activated in each treatment group. Shown are cadmium correlated genes specific for acute $(24 \mathrm{~h})$ and chronic (13 days) exposure and genes common to both treatment groups. The number of cadmium correlated genes in each group is also broken down into the number of genes induced or repressed by cadmium. 


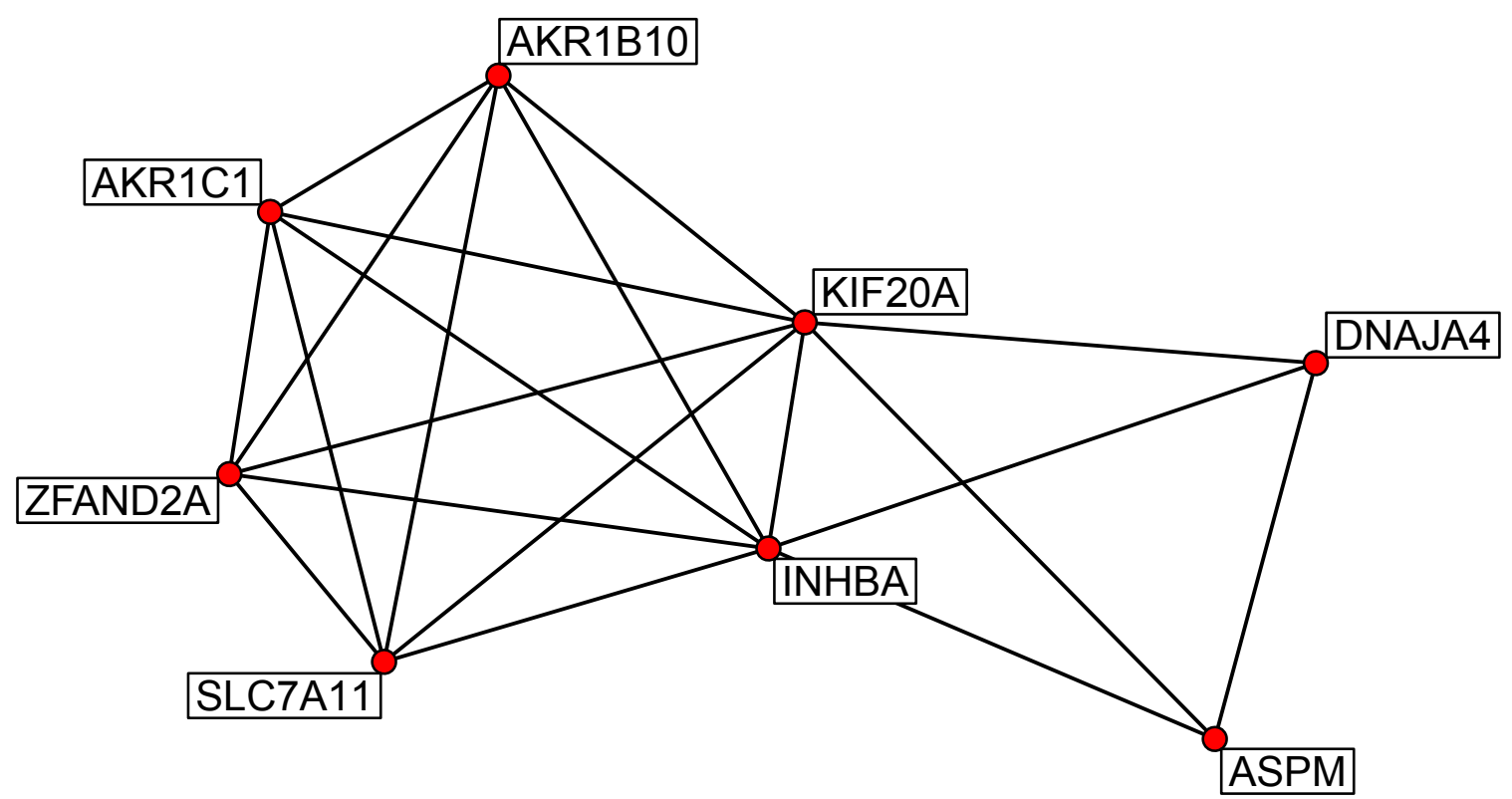

Figure 4 A network module of $\mathbf{8}$ genes that interact during cadmium toxicity. The edge between two genes indicates they are interacting directly.

variable genes across all samples. The network analysis revealed gene network modules consisting of a small group of genes that intensively interacted with each other. The network modules can be verified in molecular experiments and they serve as hypothesized models for functional relations of genes when cooperating for toxicology responses induced by cadmium.

\section{Authors' contributions}

SG, SS and DS designed and conducted the experiments. KC carried out regression analysis. YD contributed to network analysis. KZ contrived the analysis plan and developed analysis tools. KZ and SG contributed in writing the manuscript.

\section{Competing interests}

The authors declare that they have no competing interests.

\section{Acknowledgements}

This study was in part supported by grants from the National Center for Research Resources (5P2ORR016471-12) and the National Institute of General Medical Sciences (8 P20 GM103442-12) from the National Institutes of Health. The research was also supported by funds provided by the Department of Pathology and the School of Medicine and Health Sciences, University of North Dakota.

This article has been published as part of BMC Medical Genomics Volume 6 Supplement 1, 2013: Proceedings of the 2011 International Conference on Bioinformatics and Computational Biology (BIOCOMP'11). The full contents of the supplement are available online at http://www.biomedcentral.com/ bmcmedgenomics/supplements/6/S1. Publication of this supplement has been supported by the International Society of Intelligent Biological Medicine.

\section{Author details}

'Department of Pathology, School of Medicine and Health Sciences, University of North Dakota, Grand Forks, ND 58202, USA. ${ }^{2}$ Bioinformatics Core, School of Medicine and Health Sciences, University of North Dakota,
Grand Forks, ND 58202, USA. ${ }^{3}$ Rush University Cancer Center, Chicago, IL 60612, USA.

Published: 23 January 2013

\section{References}

1. Satarug S, et al: Cadmium, environmental exposure, and health outcomes. Environmental health perspectives 2010, 118(2):182-90.

2. Prozialeck WC, Edwards JR: Mechanisms of cadmium-induced proximal tubule injury: new insights with implications for biomonitoring and therapeutic interventions. The Journal of pharmacology and experimental therapeutics 2012, 343(1):2-12

3. Andrews GK: Regulation of metallothionein gene expression by oxidative stress and metal ions. Biochemical pharmacology 2000, 59(1):95-104.

4. Akesson A, et al: Tubular and glomerular kidney effects in Swedish women with low environmental cadmium exposure. Environmental health perspectives 2005, 113(11):1627-31.

5. Jarup $L$, et al: Low level exposure to cadmium and early kidney damage: the OSCAR study. Occupational and environmental medicine 2000, 57(10):668-72.

6. Kjellstrom T: A mathematical model for the accumulation of cadmium in human kidney cortex. Nordisk hygienisk tidskrift 1971, 52(2):111-9.

7. Sens DA, et al: Tissue culture of human renal epithelial cells using a defined serum-free growth formulation. Experimental nephrology 1999, 7(5-6):344-52.

8. Somji $\mathrm{S}$, et al: Expression of metallothionein isoform 3 (MT-3) determines the choice between apoptotic or necrotic cell death in $\mathrm{Cd}+2$-exposed human proximal tubule cells. Toxicological sciences: an official journal of the Society of Toxicology 2004, 80(2):358-66.

9. Somji $\mathrm{S}$, et al: The unique $\mathrm{N}$-terminal sequence of metallothionein-3 is required to regulate the choice between apoptotic or necrotic cell death of human proximal tubule cells exposed to $\mathrm{Cd}+2$. Toxicological sciences: an official journal of the Society of Toxicology 2006, 90(2):369-76.

10. Garrett SH, et al: Microarray analysis of gene expression patterns in human proximal tubule cells over a short and long time course of cadmium exposure. Journal of toxicology and environmental health Part A 2011, 74(1):24-42.

11. Meyer PE, Lafitte F, Bontempi G: Minet: a R/Bioconductor package for inferring large transcriptional networks using mutual information. $B M C$ Bioinformatics 2008, 9:461. 
12. Subramanian A, et al: Gene set enrichment analysis: a knowledge-based approach for interpreting genome-wide expression profiles. Proceedings of the National Academy of Sciences of the United States of America 2005, 102(43):15545-50.

13. Efron $B$, Tibshirani R: On testing the significance of sets of genes. The Annals of Applied Statistics 2007, 1(1):23.

14. Franchi $L$, et al: Nucleotide-binding oligomerization domain-like receptors: intracellular pattern recognition molecules for pathogen detection and host defense. Journal of immunology 2006, 177(6):3507-13

15. Zhang $\mathrm{K}$, et al: From genes to networks: in systematic points of view. BMC systems biology 2011, 5(Suppl 3):11.

16. Schlesinger MJ: How the cell copes with stress and the function of heat shock proteins. Pediatric research 1994, 36(1 Pt 1):1-6.

17. van de Water $B$, et al: Cellular stress responses and molecular mechanisms of nephrotoxicity. Toxicology letters 2006, 162(1):83-93.

18. Sun $Y$, MacRae TH: The small heat shock proteins and their role in human disease. The FEBS journal 2005, 272(11):2613-27.

19. Daugaard M, Rohde M, Jaattela M: The heat shock protein 70 family: Highly homologous proteins with overlapping and distinct functions. FEBS letters 2007, 581(19):3702-10.

20. Liu J, Qu W, Kadiiska MB: Role of oxidative stress in cadmium toxicity and carcinogenesis. Toxicology and applied pharmacology 2009, 238(3):209-14

21. Somji S, et al: Heat shock protein 27 expression in human proximal tubule cells exposed to lethal and sublethal concentrations of $\mathrm{CdCl} 2$. Environmental health perspectives 1999, 107(7):545-52.

22. Somji S, et al: Expression of the constitutive and inducible forms of heat shock protein 70 in human proximal tubule cells exposed to heat, sodium arsenite, and $\mathrm{CdCl}(2)$. Environmental health perspectives 1999, 107(11):887-93.

23. Somji $\mathrm{S}$, et al: Expression of heat shock protein 60 in human proximal tubule cells exposed to heat, sodium arsenite and $\mathrm{CdCl}_{2}$. Toxicology letters 2000, 115(2):127-36.

24. Somji S, et al: Expression of hsp 90 in the human kidney and in proximal tubule cells exposed to heat, sodium arsenite and cadmium chloride. Toxicology letters 2002, 133(23):241-54.

25. Verougstraete V, Lison D, Hotz P: Cadmium, lung and prostate cancer: a systematic review of recent epidemiological data. Journal of toxicology and environmental health Part B, Critical reviews 2003, 6(3):227-55.

26. Armstrong BG, Kazantzis G: Prostatic cancer and chronic respiratory and renal disease in British cadmium workers: a case control study. British journal of industrial medicine 1985, 42(8):540-5.

27. Sorahan T, Esmen NA: Lung cancer mortality in UK nickel-cadmium battery workers, 1947-2000. Occupational and environmental medicine 2004, 61(2):108-16.

28. Zhang $K$, et al: Gene set analysis for longitudinal gene expression data. BMC Bioinformatics 2011, 12:273.

29. Bathke $A C$, et al: Serires of randomized complete block experiments with non-normal data. Computational Statatistics and Data Analysis 2010, 57(7):1840-1857.

doi:10.1186/1755-8794-6-S1-S2

Cite this article as: Garrett et al:: Short and long term gene expression variation and networking in human proximal tubule cells when exposed to cadmium. BMC Medical Genomics 2013 6(Suppl 1):S2.

\section{Submit your next manuscript to BioMed Central and take full advantage of:}

- Convenient online submission

- Thorough peer review

- No space constraints or color figure charges

- Immediate publication on acceptance

- Inclusion in PubMed, CAS, Scopus and Google Scholar

- Research which is freely available for redistribution 